\title{
Cross-sectional Study of Glucose Disturbances of Pregnant Women in Bulgaria
}

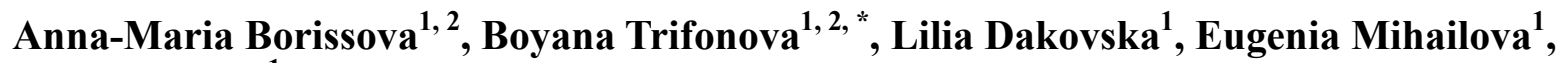 \\ Mircho Vukov ${ }^{1}$ \\ ${ }^{1}$ Clinic of Endocrinology, University Hospital Sofiamed, Sofia, Bulgaria \\ ${ }^{2}$ Faculty of Medicine, Sofia University St. Kliment Ohridski, Sofia, Bulgaria
}

Email address:

boianatri@abv.bg (B. Trifonova)

${ }^{*}$ Corresponding author

\section{To cite this article:}

Anna-Maria Borissova, Boyana Trifonova, Lilia Dakovska, Eugenia Mihailova, Mircho Vukov. Cross-sectional Study of Glucose Disturbances of Pregnant Women in Bulgaria. Clinical Medicine Research. Vol. 10, No. 1, 2021, pp. 9-15. doi: 10.11648/j.cmr.20211001.12

Received: December 16, 2020; Accepted: December 23, 2020; Published: January 18, 2021

\begin{abstract}
Hyperglycemia is one of the most common conditions during pregnancy. The International Diabetes Federation (IDF) estimates that one in six live births (16.8\%) are in women with some form of Hyperglycemia during pregnancy. The aim is to analyze the frequency of Hyperglycemia during pregnancy through a cross-sectional multicenter population-based study in 84 settlements in Bulgaria, as well as to study the dynamics of this frequency during pregnancy. Material: We studied 547 pregnant women with a mean age of $30 \pm 5$ years, divided into two groups - up to 24 gestational week - g.w. (n-386, 70.6\%) and after 24 g.w. (n-161, 29.4\%). Methods: BMI before pregnancy and the current one at the time of the study were calculated. A two-hour, $75 \mathrm{~g}$ oral glucose tolerance test (OGTT) was performed. Plasma glucose was quantitatively determined using enzymatic reference method with hexokinase (Roche reagent) on Cobas e501 analyzer, in one Central laboratory on the day of the blood sampling. The results were in mmol / 1. The statistical analysis was performed using standard SPSS 13.0 for Windows. Results: Hyperglycemia was observed in 79 (14.4\%) pregnant women after fasting state or in the course of classic OGTT, according to the criteria of WHO'2019, FIGO'2015, NICE'2015, and in the remaining 468 (85.6\%) pregnant women - Normoglycemia. There was no significant difference in the level of glycemia between the three trimesters for each of the points of OGTT $(0,60,120$ minutes) in the subgroups of pregnant women with Hyperglycemia (up to 12 g.w, 13-24 g.w, after 24 g.w.). It turned out that the number and frequency of pregnant women with Hyperglycemia in the period up to 24 g.w. is $7.5 \%(29 / 386)$ and increases in the period after 24 g.w. reaching $31 \%(50 / 161), \mathrm{P}<0.01$. According to the generally accepted population diagnostic criteria for diabetes in non-pregnant women, we found that $8.9 \%$ (7/79) of pregnant women with Hyperglycemia had Diabetes in Pregnancy (DIP), all of whom had a risk factor for Diabetes (two of them even 3 risk factors). Conclusion: As soon as pregnancy is established, verbal screening for diabetes risk factors should be performed as a first step in selecting pregnant women for glucose tolerance screening with classic OGTT to determine the glucose status of the pregnant woman.
\end{abstract}

Keywords: Pregnancy, Verbal Screening, Hyperglycemia, Gestational Diabetes Mellitus, Diabetes in Pregnancy

\section{Introduction}

In 1960, O'Sullivan et al. first demonstrated that screening, diagnosis and treatment of Hyperglycemia in pregnant women not previously known to have Diabetes mellitus, improve the outcome of the current pregnancy [1].

Hyperglycemia is one of the most common conditions during pregnancy. The International Diabetes Federation (IDF) estimates that one in six live births $(16.8 \%)$ are from women with some form of Hyperglycemia during pregnancy [2]. Gestational diabetes mellitus (GDM) affected approximately $12.8 \%$ of pregnancies worldwide in 2019 , according to the estimation of the IDF [3], and Cho NH. et al. (2018) estimates that worldwide GDM affects 18.4 million pregnant women annually [4].

According to the classification of the World Health Organization (WHO) from 2019 [5], Hyperglycemia detected for first time during pregnancy may be related to:

1. Diabetes mellitus (type 1 or type 2 ) first diagnosed 
during pregnancy according to generally accepted population diagnostic criteria for diabetes (standard OGTT 75 g glucose): fasting plasma glucose $\geq 7.0 \mathrm{mmol}$ / L or plasma glucose at 2 hours $\geq 11.1 \mathrm{mmol} / \mathrm{L}$ or $\mathrm{HbA} 1 \mathrm{c} \geq 48 \mathrm{mmol} / \mathrm{mol}$ or $\geq 6.5 \%$.

2. Gestational diabetes - hyperglycaemia above the diagnostic threshold for gestational diabetes: fasting plasma glucose $5.1-6.9 \mathrm{mmol} / \mathrm{L}$, plasma glucose at 1 hour $\geq 10.0 \mathrm{mmol} / \mathrm{L}$, plasma glucose at 2 hours 8.5 $11.0 \mathrm{mmol} / \mathrm{L}$.

These criteria are also shared by International Federation of Gynecology and Obstetrics (FIGO) [6].

In 2015, the National Institute for Health and Care Excellence (NICE) published the following criteria for diagnosing GDM [7]:

1. Plasma Fasting Blood Glucose $(\mathrm{PFBG}) \geq 5.6 \mathrm{mmol} / \mathrm{L}$ or

2. Plasma Blood Glucose at $120 \mathrm{~min}$. $\geq 7.8 \mathrm{mmol} / \mathrm{L}$

The NICE Recommendations instruct that an oral glucose tolerance test (OGTT) must be performed between $10-16$ gestational week (g.w.) to detect undiagnosed Diabetes mellitus. Additionally, in case of normal results in the first screening, OGTT is performed in $24-28$ g.w. to detect the GDM. Pregnant women who have risk factors for GDM are screened, namely:

1) $\mathrm{BMI} \geq 30 \mathrm{~kg} / \mathrm{m}^{2}$

2) Previous macrosomic baby weighing $4.5 \mathrm{~kg}$ or above

3) GDM in a previous pregnancy

4) History of diabetes mellitus in first degree relatives

Women with GDM in previous pregnancies should self-monitor blood sugar or as soon as pregnancy is diagnosed, an OGTT is performed (first or second trimester) and again OGTT in $24-28$ g.w, if the result of the first OGTT is normal. The accepted diagnostic criteria for GDM are shared by both NICE and FIGO [6].

For pregnant women with already diagnosed Diabetes, it is recommended that capillary blood glucose to be fasted at 5.3 $\mathrm{mmol} / 1$, one hour after a meal $-7.8 \mathrm{mmol} / 1$ and two hours after a meal - $6.4 \mathrm{mmol} / \mathrm{L}$. Strictly individualizes the target of blood sugar in the self-monitoring of capillary blood during pregnancy, taking into account the risk of hypoglycemia [7].

After specifying the disturbances of glucose tolerance, a certain algorithm is followed and the first step should be the introduction of appropriate diet and physical activity, and only then come the possible other therapeutic interventions $[8,9]$.

The aim of the present study is, according to available diagnostic criteria, to analyze the frequency of Hyperglycemia during pregnancy, as well as to investigate the dynamics of this frequency during pregnancy (Hyperglycemia - total; Hyperglycemia before and after 24 g.w. and newly diagnosed Diabetes mellitus for the first time during pregnancy).

The study was conducted as a cross-sectional multicenter population-based study and was conducted from September 25 to November 6, 2019 in ten regions of Bulgaria (Sofia, Samokov, Pirdop, Smolyan, Gotse Delchev, Gabrovo, Troyan, Burgas, Stara Zagora, Pleven) and their adjacent towns and villages or a total of 84 settlements. We turned to 104 endocrinologists and gynecologists for cooperation, who invited more than 630 pregnant women to participate in the screening. Of these, 547 pregnant women (86.8\%) took part.

\section{Material}

We investigated 547 pregnant women, mean age $30 \pm 5$ years, median 30 years ( $\min 18-\max 47$ years) - 40\% (28 - 32 years), $23.4 \%$ (33 - 37 years), 21\% (23 - 27 years), 8.4\% (38 - 42 years, $6 \%$ (18 - 22 years) and $1.1 \%$ (43 - 47 years).

Body Mass Index (BMI) before pregnancy was $23.36 \pm 5.05$ $\mathrm{kg} / \mathrm{m}^{2}$, Median 22.06 (minimum 15.24 - maximum 63.25), and BMI at the time of screening was $25.91 \pm 5.11 \mathrm{~kg} / \mathrm{m}^{2}$, Median 25.25 (minimum 16.51 - maximum 63.25).

The examined pregnant women were distributed by trimesters as follows: first - 110 (20.1\%), second - $276(50.5 \%)$, third - 161 (29.4\%). Additionally, according to the number of gestational weeks (g.w.), pregnant women are divided into two groups before and after 24 g.w. - 386 (70.6\%) pregnant women up to the 24-th g.w. were included in group 1, and 161 (29.4\%) pregnant women after the 24-th g.w. were included in group 2.

All participants signed informed consent, confirmed by the local Ethics Commission at Sofiamed University Hospital, Sofia University "Saint Kliment Ohridski". It was prepared in accordance with the ethical standards according to the Helsinki Declaration-1964 and later additions [10]. Each pregnant woman filled in a Questionnaire with the assistance of a specially designated medical person from the "face to face" team in order to correctly collect data on pregnancy history, past healthy history, family history for diabetes, hypertension, thyroid and chronic kidney disease, current and past medication, smoking. All participants were Caucasian, with no evidence of liver or kidney disease or evidence of malabsorption.

\section{Methods}

\subsection{The Weight and Height, BMI - Before and During Pregnancy; Arterial Pressure}

After completing the personal Questionnaire, the current weight and height of each pregnant woman were measured and filled in the Questionnaire together with the weight before pregnancy. The body mass index (BMI $-\mathrm{kg} / \mathrm{m}^{2}$ ) before pregnancy and the current one at the time of the study were calculated. At addition Arterial blood pressure was measured in the sitting position after a 5-minute rest.

\subsection{Laboratory Analysis}

A two-hour, $75 \mathrm{~g}$ oral glucose tolerance test was performed. Venous blood was drawn at 0,60 and 120 minutes in plasma tubes, containing Na2EDTA and $\mathrm{NaF}$, as an inhibitor of glycolysis for stability of glucose in the samples. The samples were transported to the laboratory after centrifugation. All of the samples were analysed in one Central laboratory on the day of the blood sampling. Glucose was quantitatively determined using enzymatic reference method with hexokinase (Roche reagent) on Cobas e501 analyzer. The results were in $\mathrm{mmol} / \mathrm{L}$. Established precision using human 
samples and controls:

1) Intra assay: Level $1(n=6) C V=1.12 \%$; Level $2(n=6)$ $\mathrm{CV}=0.42 \%$

2) Inter assay: Level $1(n=30) C V=1.25 \%$; Level $2(n=30)$ $\mathrm{CV}=1.58 \%$

3) Two levels Intralaboratory quality control on a daily basis was performed.

The Laboratory participates in two EQA systems Bulgarian EQAS and INSTAND and has certificates for this parameter.

\subsection{The Statistical Analysis}

The statistical analysis was performed using standard SPSS 13.0 for Windows: descriptive statistics (mean, medians, standard deviation), correlation analysis and analysis of variance (ANOVA, post-hoc test - with Bonferroni alpha correction), using parametrical and non-parametrical methods, including - Chi-Square Test, Fisher's Exact Test, Kolmogorov-Smirnov, Shapiro-Wilk Tests, Levene's Test for Equality of Variances, Student's t-test, Kruskal-Wallis test and Mann -Whitney test. All quantitative variables were presented as mean with standard deviation, median or percentage (unless specified otherwise), p values below 0.05 were accepted as statistically significant.

\section{Results}

Fasting hyperglycemia or in the course of classic OGTT, according to the criteria of WHO (2019), FIGO (2015), NICE (2015), was diagnosed in 79 (14.4\%) pregnant women, and in the remaining $468(85.6 \%)$ pregnant women we found Normoglycemia. In Table 1 are presented the plasma glucose levels during OGTT in total and in both groups of pregnant women - with Hyperglycemia and with Normoglycemia.

Table 1. Plasma glucose levels during OGTT in a total of 547 pregnant women, as well as in both groups - with Hyperglycemia (n-79) and with Normoglycemia (n-468).

\begin{tabular}{lll}
\hline OGTT & $\begin{array}{l}\text { Plasma glucose levels } \\
(\text { Mean } \pm \text { SD) }\end{array}$ & $\begin{array}{l}\text { Median } \\
(\text { min-max) }\end{array}$ \\
\hline 0 minute & & \\
Normoglycemia $(\mathrm{n}-468)$ & $4.72 \pm 0.35^{*}$ & $4.69(3.65-5.75)$ \\
Hyperglycemia $(\mathrm{n}-79)$ & $5.39 \pm 0.76^{*}$ & $5.32(4.24-8.56)$ \\
Total (n-547) & $4.82 \pm 0.49$ & $4.74(3.65-8.56)$ \\
60 minute & & \\
Normoglycemia $(\mathrm{n}-468)$ & $7.1 \pm 1.56^{*}$ & $7.26(2.98-13.04)$ \\
Hyperglycemia $(\mathrm{n}-79)$ & $10.53 \pm 1.19^{*}$ & $10.55(5.43-13.86)$ \\
Total (n-547) & $7.59 \pm 1.96$ & $7.58(2.98-13.86)$ \\
120 minute & & $5.53(2.32-8.45)$ \\
$\begin{array}{l}\text { Normoglycemia }(\mathrm{n}-468) \\
\text { Hyperglycemia }(\mathrm{n}-79)\end{array}$ & $5.56 \pm 1.20^{*}$ & $8.35(4.33-11.47)$ \\
Total (n-547) & $8.12 \pm 1.69^{*}$ & $5.73(2.32-11.47)$ \\
\hline
\end{tabular}

$* \mathrm{P}<0.001$ for all OGTT points - Hyperglycemia versus Normoglycemia

The glycemic curve of OGTT in pregnant women up to 24 g.w. (n-29) in total group and in the groups with Hyperglycemia and Normoglycemia of the same g.w. is shown in Table 2.
Table 2. Plasma glucose levels during OGTT in pregnant women up to 24 g.w. - total and in the groups with Hyperglycemia resp.with Normoglycemia.

\begin{tabular}{lll}
\hline Glycemia up to 24 g.w, n-29 & Mean \pm SD & Median (min-max) \\
\hline 0 minute & & \\
Normoglycemia, n-518 & $4.78 \pm 0.43^{*}$ & $4.72(3.65-7.79)$ \\
$\begin{array}{l}\text { Hyperglycemia, n-29 } \\
\text { Total, n-547 }\end{array}$ & $\begin{array}{l}5.49 \pm 0.95^{*} \\
4.82 \pm 0.50\end{array}$ & $\begin{array}{l}5.31(4.38-8.56) \\
60 \text { minute }\end{array}$ \\
$\begin{array}{l}\text { Normoglycemia, n-518 } \\
\text { Hyperglycemia, n-29 }\end{array}$ & $7,46 \pm 1.87^{*}$ & $7.52(2.98-13.79)$ \\
Total, n-547 & $10,06 \pm 1.95^{*}$ & $10.24(5.43-13.86)$ \\
120 minute & $7,60 \pm 1.97$ & $7.58(2.98-13.86)$ \\
Normoglycemia, n-518 & & \\
Hyperglycemia, n-29 & $5.80 \pm 1.48^{*}$ & $5.65(2.32-11.37)$ \\
Total, n-547 & $7.99 \pm 1.67 *$ & $8.19(4.40-11.47)$ \\
\hline
\end{tabular}

$* \mathrm{P}<0.001$

The glycemic curve of OGTT in pregnant women after 24 g.w. $(n-50)$ is presented in total group and in the groups with Hyperglycemia and Normoglycemia of the same g.w, Table 3.

Table 3. Plasma glucose levels during OGTT in pregnant women after 24 g.w. in total group and in the groups with Hyperglycemia resp. with Normoglycemia.

\begin{tabular}{lll}
\hline Glycemia after $\mathbf{2 4}$ g.w, n-50 & Mean \pm SD & Median (min-max) \\
\hline 0 minute & & \\
Normoglycemia,n-497 & $4.77 \pm 0.45^{*}$ & $4.72(3.65-8.56)$ \\
Hyperglycemia, n-50 & $5.34 \pm 0.63^{*}$ & $5.32(4.24-7.79)$ \\
Total, n-547 & $4.82 \pm 0.50$ & $4.74(3.65-8.56)$ \\
60 minute & & \\
Normoglycemia, n-497 & $7.28 \pm 1.73^{*}$ & $7.38(2.98-13.86)$ \\
Hyperglycemia, n-50 & $10.79 \pm 1.1^{*}$ & $10.56(7.54-13.79)$ \\
Total, n-547 & $7.60 \pm 1.97$ & $7.58(2.98-13.86)$ \\
120 minute & & \\
Normoglycemia, n-497 & $5.69 \pm 1.36^{*}$ & $5.62(2.32-11.47)$ \\
Hyperglycemia, n-50 & $8.20 \pm 1.70^{*}$ & $8.63(4.33-11.37)$ \\
Total, n-547 & $5.92 \pm 1.57$ & $5.73(2.32-11.47)$ \\
\hline
\end{tabular}

$* \mathrm{P}<0.001$

Table 4. Plasma glucose level at 0, 60 and 120 minutes in the course of OGTT up to 12 g.w., 13-24 g.w. and after 24 g.w. in 79 pregnant women with Hyperglycemia.

\begin{tabular}{llll}
\hline Groups & 0 min & 60 min & 120 min \\
\hline First trimester (up to 12 g.w.) n-11 & & & \\
mean \pm SD & $5.42 \pm 1.10$ & $10.21 \pm 1.31$ & $8.46 \pm 1.66$ \\
95\% CI for Mean & $4.68-6.17$ & $9.33-11.09$ & $7.35-9.58$ \\
Min - Max & $4.65-8.56$ & $6.85-11.72$ & $4.66-11.41$ \\
Second trimester (13-24 g.w.) n-18 & & & \\
mean \pm SD & $5.34 \pm 0.74$ & $10.27 \pm 1.70$ & $7.91 \pm 1.76$ \\
95\% CI for Mean & $5.08-5.60$ & $9.67-10.86$ & $7.30-8.53$ \\
Min - Max & $4.24-8.09$ & $5.43-13.86$ & $4.39-11.47$ \\
Third trimester (after 24 g.w.) n-50 & & & \\
mean \pm SD & $5.43 \pm 0.66$ & $10.88 \pm 1.34$ & $8.21 \pm 1.64$ \\
95\% CI for Mean & $5.20-5.66$ & $10.41-11.34$ & $7.64-8.78$ \\
Min - Max & $4.4-7.79$ & $7.54-13.79$ & $4.33-11.50$ \\
Total (n-79) & & & \\
mean \pm SD & $5.39 \pm 0.76$ & $10.52 \pm 1.52$ & $8.12 \pm 1.68$ \\
95\% CI for Mean & $5.22-5.56$ & $10.18-10.8$ & $7.74-8.50$ \\
Min - Max & $4.24-8.56$ & $5.43-11.86$ & $4.33-11.47$ \\
\hline
\end{tabular}

No significant difference in glycemia was found between the three trimesters for each of the OGTT points $(0,60,120$ minutes) in the groups of pregnant women with Hyperglycemia (up to 12 g.w, 13-24 g.w, after 24 g.w/), table 4. 
The analysis of the group of 79 (14.4\%) pregnant women with Hyperglycemia distributed according to the term of their pregnancy - before and after 24 g.w. showed that the frequency of the distribution almost doubled after 24 g.w. $(\mathrm{P}<$ $0.01)$, relative to the total number of 547 screened pregnant women, Table 5.

Table 5. Pregnant women (number and percentage) with Hyperglycemia total, before and after 24 g.w. in all 547 pregnant women studied, according to the criteria of WHO, FIGO, NICE.

\begin{tabular}{llll}
\hline Hyperglycemia & Total & Up to 24 g.w. & After 24 g.w. \\
\hline Yes & $79(14.4 \%)$ & $29(5.3 \%)$ & $50(9.1 \%)$ \\
No & $468(85.6 \%)$ & $518(94.7 \%)$ & $497(90.9 \%)$ \\
Total & $547(100 \%)$ & $547(100 \%)$ & $547(100 \%)$ \\
\hline
\end{tabular}

It turned out that the number and frequency of pregnant women with Hyperglycemia in the period up to 24 g.w. is $7.5 \%$ $(29 / 386)$ and increases in the period after 24 g.w. reaching $31 \%$ $(50 / 161), \mathrm{P}<0.01$. There is a fourfold increase in frequency.

Finally, additional analysis showed that Hyperglycemia was observed in $38 \%(11 / 29)$ of pregnant women up to $12 \mathrm{~g}$.w. and it reaches $62 \%(18 / 29)$ - in the period $13-24$ g.w. or almost doubling.

In the following table 6 we present the level of glycemia during OGTT of pregnant women in the subgroup - up to 24 g.w. and in subgroups - up to 12 g.w. and 13-24 g.w, with no significant difference in any of the values (NS).

Table 6. Plasma glucose level at 0, 60, 120 minutes of OGTT in 29 pregnant women with Hyperglycemia in two subgroups - up to 12 g.w.versus 13-24 g.w.

\begin{tabular}{llll}
\hline Gestational week & $\begin{array}{l}\text { Up to 12 g.w, } \\
\mathbf{1 1} / \mathbf{2 9}(\mathbf{3 7 . 9 \% )}\end{array}$ & $\begin{array}{l}\mathbf{1 3 - 2 4} \text { g.w, } \\
\mathbf{1 8} / \mathbf{2 9}(\mathbf{6 2 . 1 \%})\end{array}$ & $\begin{array}{l}\text { Total up to 24 } \\
\text { g.w. 29 (100\%) }\end{array}$ \\
\hline OGTT -0 minute & & & \\
Mean \pm SD & $5.43 \pm 1.17$ & $5.51 \pm 0.87$ & $5.49 \pm 0.95$ \\
95\% CI for Mean & $4.59-6.26$ & $5.08-5.94$ & $5.13-5.85$ \\
Min-Max & $4.65-8.56$ & $4.38-8.09$ & $4.38-8.56$ \\
OGTT -60 minute & & & \\
Mean \pm SD & $10.22 \pm 1.38$ & $10.04 \pm 2.26$ & $10.06 \pm 1.95$ \\
95\% CI for Mean & $9.23-11.21$ & $8.91-11.16$ & $9.31-10.80$ \\
Min-Max & $6.85-11.72$ & $5.43-13.86$ & $5.43-13.86$ \\
OGTT -120 minute & & & \\
Mean \pm SD & $8.51 \pm 1.74$ & $7.81 \pm 1.62$ & $7.99 \pm 1.67$ \\
95\% CI for Mean & $7.25-9.75$ & $7.01-8.61$ & $7.35-8.62$ \\
Min-Max & $4.66-11.41$ & $4.40-11.47$ & $4.40-11.47$ \\
\hline
\end{tabular}

It should be emphasized that there is no significant difference in glycemic values when comparing each point of OGTT between pregnant women up to 24 g.w. and those after 24 g.w. (Table 2 and Table 3), NS.

We applied the generally accepted population diagnostic criteria for diabetes in non-pregnant women and found that only $1.28 \%$ (7/547) pregnant women met them (WHO'2019, NICE'2015, FIGO'2015). In this small group of pregnant women, there is no evidence of previous GDM or Elevated blood sugar before pregnancy, and there is no evidence of antidiabetic treatment. Prior to pregnancy, $57 \%$ of women were obese or overweight. A history of diabetes mellitus (HD) in first degree relatives was found in only one pregnant woman and in another - a previous macrosomic baby weighing $\geq 4.5 \mathrm{~kg}$. Four of these pregnant women (57\%) had a known thyroid disease for which they were taking Levothyroxine. Table 7 presents the characteristics of these 7 pregnant women.

Table 7. Characteristics of the 7 pregnant women with newly diagnosed diabetes mellitus.

\begin{tabular}{|c|c|c|c|c|c|c|c|}
\hline Code & 30 & 115 & 377 & 217 & 442 & 525 & 539 \\
\hline Sequence pregnancy & 1 & 1 & 1 & 3 & 2 & 1 & 4 \\
\hline Gestational week & 13 & 17 & 36 & 23 & 31 & 13 & 15 \\
\hline $\begin{array}{l}\text { Increased blood glucose } \\
\text { before pregnancy }\end{array}$ & No & No & No & No & No & No & No \\
\hline Previous GDM & No & No & No & No & No & No & No \\
\hline Known DM & No & No & No & No & No & No & No \\
\hline Treatment for DM & No & No & No & No & No & No & No \\
\hline BMI before pregnancy & 30.4 & 37.3 & 19.1 & 23.8 & 25.3 & 23.8 & 28.8 \\
\hline BMI at screening & 32.2 & 39.2 & 27.7 & 25.7 & 29.3 & 26.0 & 28.9 \\
\hline $\begin{array}{l}\text { A previous macrosomic } \\
\text { baby } \geq 4.5 \mathrm{~kg} \text { or a } \mathrm{HD}\end{array}$ & No & $\begin{array}{l}\text { Yes } \\
\text { HD }\end{array}$ & No & $\begin{array}{l}\text { Yes } \geq \\
4 \mathrm{~kg}\end{array}$ & No & No & No \\
\hline Thyroid disease & No & Yes & Yes & Yes & No & Yes & No \\
\hline $\begin{array}{l}\text { Treatment with Levothyro } \\
\text { xin }\end{array}$ & No & Yes & Yes & Yes & No & Yes & No \\
\hline
\end{tabular}

Table 8 presents the data from OGTT in these 7 women with newly diagnosed Diabetes mellitus ie. Diabetes in Pregnancy (DIP) at different stages of their pregnancy - from 13 g.w. to 36 g.w.

Table 8. Plasma glucose level during OGTT - 0, 60, 120 minutes in 7 pregnant women with newly diagnosed Diabetes in Pregnancy.

\begin{tabular}{llll}
\hline Code & 0 minute & 60 minute & 120 minute \\
\hline $30 *$ & 8.56 & 11.03 & 8.19 \\
$115^{*}$ & 8.09 & 7.03 & 4.40 \\
377 & 7.79 & 9.32 & 5.39 \\
$217 * *$ & 5.42 & 11.91 & 11.37 \\
442 & 5.23 & 10.23 & 11.15 \\
525 & 4.81 & 11.72 & 11.41 \\
$539 * *$ & 4.79 & 11.10 & 11.47 \\
\hline
\end{tabular}

*BMI - $\geq 30 \mathrm{~kg} / \mathrm{m}^{2} ; * * \mathrm{BMI}-25.00-29.00 \mathrm{~kg} / \mathrm{m}^{2}$ (before pregnancy)

\section{Discussion}

In the Bulgarian Recommendations on Diabetes from 2019 of Bulgarian Society of Endocrinology (BSE) [11] a special section is dedicated to Screening for Gestational Diabetes. It is recommended that OGTT be performed in women at increased risk of developing diabetes at the first prenatal consultation, using the usual WHO criteria for the diagnosis of diabetes. Screening for Diabetes mellitus with OGTT is performed during 24 - 28 weeks of pregnancy in all pregnant women who have not been diagnosed with Diabetes mellitus in early pregnancy, with a plasma glucose test.

Following the already mentioned criteria of the international organizations WHO, NICE, FIGO, as well as those of BSE for assessment of glucose tolerance disorders in the course of OGTT in pregnant women, we divided and analyzed our 547 pregnant women in two main groups - with Hyperglycemia (n-79) and with Normoglycemia (n-468). Our incidence of Hyperglycemia of $14.4 \%$ during pregnancy is within the expected range and presented in various studies. 
The International Diabetes Federation states that about $15 \%$ of pregnant women in the world develop GDM during pregnancy and this frequency varies in different parts of the world [12]. In our material, when comparing each point of the glycemia curves in the course of OGTT in pregnant women, those with Hyperglycemia differed significantly from the group of pregnant women with Normoglycemia $(\mathrm{P}<0.001)$, Table 1 .

When comparing the glycemia curve of OGTT in pregnant women in the two major groups - before and after 24 g.w., in the groups with Hyperglycemia and Normoglycemia of the same gestational week, the differences were also significant ( $P$ $<0.001$ ), Table 2 and Table 3.

In addition, comparisons of these curves were made only for the group of pregnant women with Hyperglycemia for the three periods of pregnancy - up to 12 g.w, 13-23 g.w. and after 24 g.w. finding no significant difference (NS), Table 4. Therefore, glycemic disorders are present in all stages of pregnancy and they are of identical severity. For us, the analysis of the frequency of Hyperglycemia, which is a common disorder during pregnancy, was much more important and was analyzed in detail, as in the three periods of pregnancy - up to 12 g.w, 13-24 g.w. and after 24 g.w, and in both major periods before and after 24 g.w.

During the first period up to 24 g.w. we identified 29 pregnant women with Hyperglycemia versus 50 pregnant women with Hyperglycemia in the next period after 24 g.w. This means that up to 24 g.w. the incidence of Hyperglycaemia was $5.3 \%$ (29/547) compared to a total of 547 women studied, and after 24 g.w. - 9.1\% (50/547), P < 0.01 or there is almost a double increase during pregnancy.

It turned out, that the number and frequency of pregnant women with Hyperglycemia increased significantly during pregnancy compared to each period - from 7.5\% (29/386) up to 24 g.w. the frequency reached $31 \%(50 / 161)$ after 24 g.w, P $<0.01$. In fact, there is a fourfold increase in the incidence of the disorder after 24 g.w. and screening for dysglycemia is particularly important at this period.

This phenomenon of increase in the incidence of cases of Hyperglycemia during pregnancy can be seen in the comparison within each of the small periods up to $12 \mathrm{~g}$.w. and 13-24 g.w, when a total of 29 pregnant women with Hyperglycaemia were identified for these two 12-week periods.

There is almost a double increase - from 38\% (11/29) for the subgroup of pregnant women up to 12 g.w. and reached to $62 \%$ $(18 / 29)$ for the subgroup of pregnant women 13-24 g.w. It should be noted that the level of glycemia during OGTT of pregnant women in both subgroups - up to 12 g.w. and 13-24 g.w, showed no significant difference at any of the points (NS). Therefore, the severity of the disturbance of glycemic control is present in the first 12 g.w. and is identical for the two early periods of pregnancy - up to 12 g.w. and 13-24 g.w.

The prevalence of pregestational and gestational diabetes is increasing worldwide. In pregnancies complicated by Diabetes, placental dysfunction and hormonal changes can occur, leading to a number of complications [13].

Normal pregnancy is associated with the development of insulin resistance, especially from the second trimester, and is similar to that found in type 2 diabetes. These changes facilitate glucose transport through the placenta to ensure normal fetal growth and development $[1,14,15]$. If maternal gestational insulin resistance becomes too pronounced, then maternal GDM may be diagnosed. Traditionally, GDM is defined as any Hyperglycemia that is first identified during pregnancy, including pre-existing undiagnosed diabetes / hyperglycemia [16].

We strictly followed the diagnostic algorithm and interpretation of the results according to the criteria in the recommendations of established international organizations $[17,18]$. Thus, we diagnosed 7 (1.28\%) pregnant women with Diabetes in Pregnancy (DIP). According to the International Diabetes Federation (2019), about $16.8 \%$ of women have some form of Hyperglycemia during pregnancy. About $16 \%$ of them have Pre-pregnancy Diabetes or Diabetes in Pregnancy, and $84 \%$ have GDM [2]. In our material of all 79 pregnant women with Hyperglycemia in $8.9 \%(7 / 79)$ of them refers to Diabetes in Pregnancy (DIP). It should be emphasized that these pregnant women have newly diagnosed Diabetes mellitus first established during pregnancy, according to generally accepted population diagnostic criteria for diabetes in non-pregnant (standard OGTT $75 \mathrm{~g}$ glucose).

In these pregnant women, obesity / overweight is present in $57 \%$ of cases. During pregnancy, obese women became more obese and all other pregnant women became overweight. None of this group of pregnant women was left with a normal BMI during their pregnancy. Hypothyroidism treated with Levothyroxin diagnosed before pregnancy had $57 \%$ of these pregnant women, and in two of the pregnant women this was the third risk factor for diabetes. Of the other risk factors, there was only in one case with previous macrosomic baby weighing $4.5 \mathrm{~kg}$ or above and in another with history of diabetes mellitus in first degree relatives.

It should be noted that the diagnosis of diabetes in these pregnant women was made for the first time at this point in their pregnancy, although five of them had one risk factor and two pregnant women had three risk factors. Therefore, all pregnant women in this group had risk factors for diabetes, but missed prenatal screening. This OGTT is performed in: 2 pregnant women - on 13 g.w., 2 pregnant women - on 15-17 g.w. and the other 3 pregnant women - after 31 g.w. and it is too late to diagnose impaired glucose tolerance. And all this is allowed, although for at least 15 years the importance of pre-conception intervention is emphasized, because $30-90 \%$ of women have at least one risk factor - anemia, malnutrition, obesity, diabetes, hypertension, thyroid disorders. Preliminary diagnosis and correction of the respective disorders would make the subsequent desired pregnancy successful [19].

\section{Conclusion}

In the studied population of pregnant women in $14.4 \%$ Hyperglycemia was found, as $5.3 \%$ of the cases were up to 24 g.w. and $9.1 \%$ - after 24 g.w. Therefore, it is imperative that a classic OGTT be performed as soon as pregnancy is established to determine the glucose status of the pregnant 
woman. Although the percentage of cases of Diabetes in pregnancy (DIP) is too small, its timely diagnosis is extremely important. In this small group of 7 pregnant women in our material there is one risk factor for diabetes, and in two of them - even three risk factors. This indicates that verbal screening is a useful first step in selecting pregnant women for glucose tolerance screening. Thus, it is common to perform the classic OGTT to identify not only the possible presence of Gestational diabetes mellitus (GDM), but also the much rarer Diabetes in pregnancy (DIP) according to the relevant diagnostic criteria. All this is in the interest of the pregnant woman, the fetus and society, in order to create and give birth to a healthy generation.

In this regard, the need to conduct a follow-up study on the same group of surveyed women should be emphasized, as the current project was a cross-sectional study. Thus, early detection of Diabetes in Pregnancy (DIP) will ensure timely and complete treatment of women with newly diagnosed diabetes, which may determine their future. Pregnant women with GDM must be monitored by their personal General practitioners/Endocrinologists according to the rules of consensus and standards of International Diabetes organizations, as well as our National association.

\section{Conflicts of Interests}

All the authors do not have any possible conflicts of interest.

\section{Acknowledgements}

The authors thank the endocrinologists and obstetrician/gynecologists who provided Local support: V. Jotova (Troyan), S. Dimitrova (Gabrovo), Anchev (Gabrovo), Mitev (Gabrovo), A. Popov (Goce Delchev), A. Andreev (Samokov), E. Apostolova (Smolyan), M. Hubshev (Smolyan), S. Dimitrov (Pirdop), R. Naumovska (Pleven), M. Manuelyan (Burgas), K. Kirovakov (Burgas), T. Gabrovska (Burgas), K. Venkova (Sofia), M. Angelova (Stara Zagora), R. Velev (Sofia), I. Sigridov (Sofia), B. Bogoslovova (Sofia) ... total 104 specialists in the country. Technical support: T. Kornilova (Sofia), Z. Metodieva (Sofia), J. Georgieva (Sofia), H. Atanasova (Burgas), I. Ilieva (Stara Zagora).

This work was supported by grants from the Bulgarian Society of Endocrinology (Project Number 7/2019) as part of the National Epidemiological Program for Pregnant Women in Bulgaria, 2019.

\section{References}

[1] O'Sullivan JB, Mahan CM. (1964). Criteria for the oral glucose tolerance test in pregnancy. Diabetes 13:278-285.

[2] International Diabetes Federation. (2019). IDF Diabetes Atlas, $9^{\text {th }}$ edn. Brussels, Belgium: International Diabetes Federation.

[3] Yuen L, Saeedi P, Riaz M, Karuranga S, Divakar H, Levitt N, Yang X, Simmons D. (2019). Projections of the prevalence of hyperglycaemia in pregnancy in 2019 and beyond: results from the International Diabetes Federation Diabetes Atlas, $9^{\text {th }}$ edition. Diabetes Res Clin Pract 157: 107841. https://doi.org/10.1016/j. diabres.2019.107841.

[4] Cho NH, Shaw JE, Karuranga S, Huang Y, da Rocha Fernandes J. D, Ohlrogge A. W, Malanda B. (2018). IDF diabetes atlas: global estimates of diabetes prevalence for 2017 and projections for 2045. Diabetes Res Clin Pract 138: 271-328.

[5] World Health Organization. (2019). Classification of Diabetes mellitus 2019. ISBN 978-92-4-151570-2.

[6] Hod M, Kapur A, Sacks D. A, Hadar E, Agarwal M, Di Renzo G. C, Roura L. C, McIntyre H. D, Morris J. L, Divakar H. The International Federation of Gynecology and Obstetrics. (2015). Initiative on gestational diabetes mellitus: A pragmatic guide for diagnosis, management, and care. International Journal of Gynecology and Obstetrics 131, S173-S211.

[7] NICE (2015). Diabetes in pregnancy: management from preconception to the postnatal period. https://www.nice.org.uk/terms-and conditions\#notice-ofrights).

[8] Farrar D, Simmonds M, Bryant M, Sheldon T. A, Tuffnell D, Golder S, Lawlor D. A. (2017). Treatments for gestational diabetes: a systematic review and meta-analysis. BMJ Open 7 (6): e015557. https://doi.org/10.1136/bmjopen-2016-015557.

[9] Bogdanet D, Reddin C, Macken E, Griffin T. P, Narjes Fhelelboom, Linda Biesty, Shakila Thangaratinam, Dempsey E, Crowther C, Galjaard S, Maresh M, Loeken M. R, Napoli A, Anastasiou E, Noctor E, de Valk H. W, van Poppe M. N. M, Agostini A, Clarson C, Egan A. M, O'Shea P. M, Devane D, Dunne F. P. (2019). Follow-up at 1 year and beyond of women with gestational diabetes treated with insulin and/or oral glucose-lowering agents: a core outcome set using a Delphi survey. Diabetologia 62: 2007-2016.

[10] World Medical Association (2013). "Declaration of Helsinki: Ethical Principles for Medical Research Involving Human Subjects". JAMA. 310 (20): 2191-2194. doi: 10.1001/jama.2013.28105.

[11] Bulgarian Society of Endocrinology. (2019). Recommendations for good clinical practice in diabetes mellitus, edited by Prof. Borisova A-M, Simolini Publishing House, Sofia, Bulgaria.

[12] International Diabetes Federation. (2011). Diabetes in pregnancy Protecting Maternal Health, Policy Briefing. Sept. 2011.

[13] McCance D, Maresh M, Sacks DA. A practical manual of diabetes in pregnancy. John Wiley \& Sons, 2017. doi: $10.1002 / 9781119043805$.

[14] Hurwitz D, Jensen DN. (1946). Carbohydrate metabolism in normal pregnancy. N Engl J Med 234 (10): 327-329. https://doi.org/10.1056/NEJM194603072341004.

[15] Lawlor DA. (2013). The Society for Social Medicine John Pemberton Lecture 2011. Developmental overnutrition-an old hypothesis with new importance? Int J Epidemiol 42 (1): 7-29. https://doi.org/10.1093/ije/dys209.

[16] Elliott H. R. \& Sharp G. S. \& Relton C. L. \& Lawlor D. A. (2019). Epigenetics and gestational diabetes: a review of epigenetic epidemiology studies and their use to explore epigenetic mediation and improve prediction. Diabetologia 62: 2171-2178. 
[17] IDF (2015). GDM model of Care. Implementation Protocol Guidelines for Healthcare Professionals.

[18] International Association of Diabetes and Pregnancy Study Groups Consensus Panel. Metzger BE, Gabbe SG, Persson B, Buchanan TA, Catalano PA, Damm P, Dyer AR, Leiva Ad, Hod M, Kitzmiler JL, Lowe LP, McIntyre HD, Oats JJ, Omori Y, Schmidt MI. (2010). International association of diabetes and pregnancy study groups recommendations on the diagnosis and classification of hyperglycemia in pregnancy. Diabetes Care 33 (3): 676-682.

[19] Kim C, Ferrara A, McEwen LN, Marrero DG, Gerzoff RB, Herman WH, TRIAD Study Group. (2005). Preconception care in managed care: the translating research into action for diabetes study. Am J Obstet Gynecol. 192 (1): 227-232. 